\title{
Fire monitoring in coal mines using wireless underground sensor network and interval type-2 fuzzy logic controller
}

\author{
Sweta Basu ${ }^{1}$ - Sutapa Pramanik ${ }^{2} \cdot$ Sanghamitra Dey $^{3} \cdot$ Gautam Panigrahi $^{3}$. \\ Dipak Kumar Jana ${ }^{4}$ D
}

Received: 12 October 2018/Revised: 28 December 2018/Accepted: 7 March 2019/Published online: 1 April 2019

(C) The Author(s) 2019

\begin{abstract}
From the view of underground coal mining safety system, it is extremely important to continuous monitoring of coal mines for the prompt detection of fires or related problems inspite of its uncertainty and imprecise characteristics. Therefore, evaluation and inferring the data perfectly to prevent fire related accidental risk in underground coal mining (UMC) system are very necessary. In the present article, we have proposed a novel type-2 fuzzy logic system (T2FLS) for the prediction of fire intensity and its risk assessment for risk reduction in an underground coal mine. Recently, for the observation of underground coal mines, wireless underground sensor network (WUSN) are being concerned frequently. To implement this technique IT2FLS, main functional components are sensor nodes which are installed in coal mines to accumulate different imprecise environmental data like, temperature, relative humidity, different gas concentrations etc. and these are sent to a base station which is connected to the ground observation system through network. In the present context, a WUSN based fire monitoring system is developed using fuzzy logic approach to enhance the consistency in decision making system to improve the risk chances of fire during coal mining. We have taken Mamdani IT2FLS as fuzzy model on coal mine monitoring data to consider real-time decision making (DM). It is predicted from the simulated results that the recommended system is highly acceptable and amenable in the case of fire hazard safety with compared to the wired and off-line monitoring system for UMC. Legitimacy of the suggested model is prepared using statistical analysis and multiple linear regression analysis.
\end{abstract}

Keywords Type-2 fuzzy logic · Underground coal mining system · Mine environment · Fire and risk monitoring of mine · Wireless sensor networks · Fire Intensity

\section{Introduction}

The productivity, quality and safety performance of an UMC systems are highly influenced by the environmental states (uncertain or fuzzy) of it. Therefore, it is necessary to observe

Dipak Kumar Jana

dipakjana@hithaldia.in

Sweta Basu

swetabasu2009@gmail.com

Sutapa Pramanik

sutapapramanik12@gmail.com

Sanghamitra Dey

iamsangha@gmail.com

Gautam Panigrahi

panigrahi_goutam@rediffmail.com the complexity and perilousness of its environment in a continuous manner to ensure the safety and risk level of coal miming. At present, this particular discussed technology (WSN) is being utilized widely in any workplace imprecise environment of an industry or like UMC system in various

1 Prajnanananda Insitute of Technology \& Management, 94/2

Park Street Kol-17 Park Circus 7th point, Kolkata,

WB 700 017, India

2 Deulia Balika Vidyamandir, Kolaghat 721154, India

Department of Mathematics, NIT, Durgapur, WB, India

4 Department of Applied Science, Haldia Institute of Technology, Haldia, Purba Midnapur, WB 721657, India 
uncertain environments. Banerjee (1985) has developed a spontaneous combustion process driven by auto-oxidation of coal with various carbonaceous matter which is basically a complex physico-chemical process used to generate heat energy and some polluting gases like $\mathrm{CO}, \mathrm{CO}_{2}$, higher degrees hydrocarbons etc. Bhattacharjee et al. (2012) has explained the enactment of WSN-based model which can be simulated for making a fire monitoring as well as alarm (FMA) system for the application in Bord-and-Piller coal mine panel. The proposed WSN-based coal mine consisted of Bord-and-Pillar system is skilled of not only providing real-time monitoring and alarm in case of a fire, but also providing the exact fire location and spreading track by continuously gathering, analyzing, and storing real time information.

Cheng et al. (2015) has developed a Web-based, lightweight remote monitoring and control platform using a wireless sensor network (WSN) with the REST style to collect temperature, humidity and methane concentration data in a coal mine using sensor nodes. The authors have implemented three different scenarios for Web-based, lightweight remote monitoring and control of coal mine safety and measured and analyze the system performance.

Coal mine production environment is very multifarious. There are many uncertain in-formations existed in the gas safety evaluation process and it is also difficult to predict fire intensity in imprecise environment. Underground environmental condition of mines are mainly responsible for the productivity and safety performance of coal mining system. The major factors that influence the different uncertain environmental conditions of an underground coal mines (UCM) are, airflow, temperature, humidity, dust and gases. These operations are essentially responsible for the generation of various toxic gases like, $\mathrm{H}_{2} \mathrm{~S}, \mathrm{CO}_{2}, \mathrm{CO}$, etc. The other highly recommended hazards which are related to the underground mining system are fire and explosions, generally happened for the spontaneous combustion of coal like inflammable materials, its dust and methane mixtures, respectively. The observation of UCM environment is vastly done with the help of various monitoring devices consisting of data loggers. To facilitate the improvement of this vulnerability, online observation or monitoring system was developed in advanced places with the aim of keeping a track of the unwanted environmental conditions of the UCM. Conversely, the system used wired connection and then suffered from many flaws such as damaging chances in connection cables or components, large fault rate, defective system maintenance etc. For that reason, online monitoring system of UCM using wireless sensor network (WSN) has become a common practice now-a-days. As a result, WSN (Akyildiz and Stuntebeck 2006) has developed like a crucial technique in continuous monitoring of an industrial workplace or in an UCM. It can be recognized by involving sensor nodes in proper positions of underground mines to gather environmental data and to detect the occurrences of probable risks like fires, explosions, gas leaks or roof failures etc. (cf. Zhi-zhong et al. 2010).

Muduli et al. (2018) has developed a WSN for environmental monitoring for UCM and this investigation represented a literature part in an organized manner based on the contemporary investigations in the application of WUSN technique. Hence, UCM driven by WSN i.e. online monitoring (Rawat et al. 2014; Mishra et al. 2013) has become the very common practice. NawrockiIzabela and Kowalska (2016) have developed a type-1 fuzzy logic model for combined internal and industrial risk assessment in a coal mining. It is important to predict conditions leading to the fire hazard by proper interpretation of the imprecise data from UCM system. The Table 1 describes a comparative study in this literature of UCM.

The type-1 fuzzy logic first proposed by Zadeh (1965) for decision making, and is able to provide accurate results based on uncertain and vague information. The most popular type1 fuzzy inference models are Mamdani and Assilian (1975) and Sugeno Sugeno (1985). Generally, each model can be represented as "If antecedence then consequence". The antecedence is the same for all type-1 fuzzy models (Dey and Jana 2016), but the consequence has different forms for each model. In spite of above mentioned development has been made, we have developed the following:

(1) The IT2FLC approach is applied to compute the system output in the construction of robust WSN based fire monitoring system for mines.

(2) In T2FLC assists to trace four inputs (temperature, $\mathrm{CO}, \mathrm{CO}_{2}, \mathrm{O}_{2}$ ) and one output fire intensity in a preplanned way to build the inference train for which the system output can be forecast.

(3) The responsible qualitative factors which are involved highly for the improvement in system output, can be easily comprised in the T2F prediction model to achieve better accuracy.

(4) Legitimacy of the suggested model is prepared using statistical analysis and multiple linear regression.

(5) An autonomous system via supervised fuzzy learning under dynamic electricity prices.

(6) A robust T2FLC model is developed to evaluate the instant updates of mine fire risk chances from the UCM imprecise data.

Table 1 Comparative review on fire monitoring system in a coal mining via WUSN

\begin{tabular}{llll}
\hline References & $\begin{array}{l}\text { No. of } \\
\text { inputs }\end{array}$ & $\begin{array}{l}\text { Uncertainty/ } \\
\text { fuzzy }\end{array}$ & $\begin{array}{l}\text { Statistical } \\
\text { analysis }\end{array}$ \\
\hline Grychowski (2014) & 3 & Type-1 & $\begin{array}{l}\text { Simulation } \\
\text { Muduli et al. (2018) }\end{array}$ \\
Proposed model & 4 & Type-1 & t-Test \\
\hline
\end{tabular}


Many researchers have shown their interest to find the appropriate locations of the sensors in a UCM. Xu et al. (2012) have proposed an ecological monitoring and miner localization system for UCMs by using ZigBee network. Wireless ZigBee technology was developed to overcome the limitation of wire communication system in UCMs. Liu et al. (2010) have developed a miner GPS system in the blind areas of underground coal mines using hierarchical low-cost ZigBee network. Sensor nodes (SNs) were used at different locations in underground mine tunnel to predict the exact position of the mine personnel. Using ZigBee and GPS system, the position of mine personnel in underground mine tunnel was calculated using the following steps: (a) gateway correct the localization error due to underground tunnel interference (b) determine the location of mine personnel in underground tunnel using ZigBee nodes and send the location information to (c) compute the position of mine personnel in 3D coordinate using Global Positioning System (GPS) system (d) approximation the position of mine personnel in shade areas of underground coal mine.

In the present investigation, an IT2FLC has been developed to create global and local decisions at the sink and sensor nodes rather than ground observation centre where temperature, and other various gases mentioned before (like $\mathrm{CO}, \mathrm{CO}_{2}$ etc.) have been taken as input variables in this T2FLS to investigate the status of any UCM firing system. Mamdani T2FIS is considered to simulate the proposed model with the help of MATLAB 7.0 fuzzy logic tool box. As the expectation of the objective of this present investigation, the ultimate results after simulation verified that the $\mathrm{T} 2 \mathrm{~F}$ based monitoring system reduces the delay in decision making above the off-line system for monitoring UCM in adverse environments.

\section{Notations and abbreviations}

The following notations and abbreviations are used to describe the proposed inference model.

(1) $U M C=$ underground coal mining

(2) $W S N=$ wireless sensor network

(3) $\quad F I S=$ fuzzy inference system.

(4) $\quad M F I S=$ Mamdani fuzzy inference system.

(5) $R^{2}=$ coefficient of determination.

(6) $R M S E=$ root mean square error.

(7) $M A E=$ mean absolute error.

(8) $M A P E=$ mean absolute percentage error.

(9) $M F=$ membership function.

(10) $U C M=$ Underground coal mine.

(11) $W U S N=$ Wireless underground sensor network.

\subsection{Type-2 fuzzy sets and IT2-FLSs}

In this section, we will present briefly and a lucid manner discussion on internal structure of IT2-FLSs and T2FS. The structure of the IT2-FLS is similar to T1- FLS counterpart. However, a type-2 fuzzy set has grades of membership that are type-1 fuzzy, so it could be called a ?fuzzy fuzzy set? and thus IT2-FLS has the extra type-reduction process. Therefore, IT2-FLS can able to tackle uncertainty in a much better way, which make T2-FS ideal for productivity forecast in fire intensity prediction.

A type-2 fuzzy set expresses the non-deterministic truth degree with imprecision and uncertainty for an element that belongs to a set (cf. Castillo and Melin 2008). A type-2 fuzzy set is denoted by $\tilde{\tilde{A}}$, is characterized by a type-2 membership function $\mu_{\tilde{A}}(x, u)$ where $x \in X, \forall u \in J_{x}^{u} \subseteq$ $[0,1]$ and $0 \leq \mu_{\tilde{A}}(x, u) \leq 1$ defined in Eq. (1)

$\tilde{\tilde{A}}=\left\{\left(x, u, \mu_{\tilde{A}}(x, u)\right) \mid x \in X, \quad \forall u \in J_{x}^{u} \subseteq[0,1]\right\}$

If $\tilde{\tilde{A}}$ is fuzzy type 2 (FT2) continuous variable, it is denoted in Eq. (2)

$$
\tilde{\tilde{A}}=\left\{\int_{x \in X}\left[\int_{u \in J_{x}^{u}} f_{x}(u) / u\right] / x\right\}
$$

where double integration denotes the union of $x$ and $u$. If $A$ is FT2 discrete, then it is denoted by Eq. (3)

$$
\tilde{\tilde{A}}=\left\{\sum_{x \in X} \mu_{\tilde{\tilde{A}}}(x) / x\right\}=\left\{\sum_{i=1}^{N}\left[\sum_{k=1}^{M_{i}} f_{x_{i}}\left(u_{k}\right) / u_{i k}\right] / x_{i}\right\}
$$

where double summation denotes the union of $x$ and $u$. If $f_{x}(u)=1, \forall u \in\left[\underline{J}_{x}^{u}, \bar{J}_{x}^{u}\right] \subseteq[0,1]$, the type-2 membership function $\mu_{\tilde{\tilde{A}}}(x, u)$ is expressed by one type-1 inferior membership function, $\underline{J}_{x}^{u}=\mu_{A}(x)$ and one type-1 superior, $\bar{J}_{x}^{u}=\mu_{A}(x)$, then it is called an interval type- 2 fuzzy set denoted by Eqs. (4) and (5).

$$
\tilde{\tilde{A}}=\left\{(x, u, 1) \mid \forall x \in X, \forall u \in\left[\underline{\mu}_{A}(x), \bar{\mu}_{A}(x)\right] \subseteq[0,1]\right\}
$$

or

$$
\begin{aligned}
\tilde{\tilde{A}} & =\left\{\int_{x \in X}\left[\int_{u \in\left[\underline{J}_{x}^{u} \bar{J}_{x}^{u}\right] \subseteq[0,1]} 1 / u\right] / x\right\} \\
& =\left\{\int_{x \in X}\left[\int_{u \in\left[\underline{\mu}_{A}(x), \bar{\mu}_{A}(x)\right] \subseteq[0,1]} 1 / u\right] / x\right\}
\end{aligned}
$$


Since $\tilde{\tilde{A}}$ is a type-2 fuzzy Singleton (Castillo and Melin 2008), the membership function is denoted and defined by Eq. (6) as:

$\mu_{\tilde{\tilde{A}}}(x)= \begin{cases}1 / 1, & \text { si } x=x^{\prime} \\ 1 / 0, & \text { si } x \neq x^{\prime}\end{cases}$

Definition 1 A type-1 fuzzy set $X$ is comprised of a domain $D_{X}$ of real numbers (also called the universe of discourse of $X$ ) together with a membership function (MF) $\mu_{x}: D_{X} \rightarrow[0,1]$, i.e.

$X=\int_{D_{x}} \mu_{x}(x) / x$

Here $\int$ denotes the collection of all points $x \in D_{X}$ with associated membership grade $\mu_{x}(x)$.

Definition 2 An IT2 FS $\tilde{X}$ is characterized by its MF $\mu_{x}(x, u)$, i.e

$\tilde{X}=\int_{x \in D_{x}}\left[\int_{u \in J_{x} \subseteq[0,1]} 1 / u\right] / x$

where $x$ called the primary variable, has domain $D_{\tilde{X}}: u \in[0,1]$, called the secondary variable, has domain $J_{x} \subseteq[0,1]$ at each $x \in D_{\tilde{X}} ; J_{x}$ also can be termed as the support of secondary MF and the amplitude will be $\mu_{\tilde{X}}(x, u)$, called a secondary grade of $\tilde{X}$, equals 1 for $\forall x \in$ $D_{\tilde{X}}$ and $\forall u \in J_{x} \subseteq[0,1]$.

Generally, T2FSs $\mu_{X}(x, u)$ may be any number in $[0,1]$, and it generally varies as $\mathrm{x}$ and/or $\mathrm{u}$ vary.

Definition 3 The uncertainty about $\tilde{X}$ is suggested by the union of all its primary memberships, which is said the footprint of uncertainty (FOU) of $\tilde{X}$, i.e.,

$$
F O U(\tilde{X})=\bigcup_{\forall x \in D_{\tilde{X}}} J_{x}=\left\{(x, u): u \in J_{x} \subseteq[0,1]\right\}
$$

The size of an FOU is directly related to the uncertainty that is conveyed by an IT2 FS. So, an FOU with more area is more uncertain than one with less area.

Definition 4 The upper membership function (UMF) and lower membership function (LMF) of $\tilde{X}$ are two T1 MFs X and $\mathrm{X}$ that bound the FOU.

$J_{x}=\left[\mu_{\underline{X}}(x), \mu_{\bar{X}}(x)\right]$

Using Eq. (10), FOU ( $\tilde{X})$ can also be expressed as

$$
\operatorname{FOU}(\tilde{X})=\bigcup_{x \in D_{x}}\left[\mu_{\underline{X}}(x), \mu_{\bar{X}}(x)\right]
$$

where the mfs can be explained as the IT2-FLS that can be looked like a mapping of the inputs to the outputs which might be expressed through a function $y=\psi(x)$. If we consider the membership functions as gaussian functions having a fixed standard deviation $\sigma$, and an uncertain mean situated in $m \in\left[m_{1}, m_{2}\right]$, as given by

$\mu_{\tilde{A}(x)}=\exp \left[-\frac{1}{2}\left(\frac{x-m_{1}}{\sigma}\right)^{2}\right]$

Usually the upper membership function (UMF) and lower membership function (LMF) are generally given as well as denoted by

$\bar{\mu}_{\tilde{A}(x)}=\left\{\begin{array}{lc}\exp \left[-\frac{1}{2}\left(\frac{x-m_{1}}{\sigma}\right)^{2}\right], & \text { if } x<m_{1} \\ 1, & m_{1} \leq x \leq m_{2} \\ \exp \left[-\frac{1}{2}\left(\frac{x-m_{2}}{\sigma}\right)^{2}\right], & \text { if } x>m_{2}\end{array}\right.$

and

$\underline{\mu}_{\tilde{A}(x)}= \begin{cases}\exp \left[-\frac{1}{2}\left(\frac{x-m_{2}}{\sigma}\right)^{2}\right], & x \leq \frac{m_{1}+m_{2}}{2} \\ \exp \left[-\frac{1}{2}\left(\frac{x-m_{1}}{\sigma}\right)^{2}\right], & x>\frac{m_{1}+m_{2}}{2}\end{cases}$

Type-reducer is basically a part of the output processing block that is used to target the reduction of output type- 2 fuzzy set to type-1 output fuzzy set. The height of typereduction has the following structure as follows

$Y(x)=\left[Y_{L}(x), Y_{R}(x)\right]$

where $Y(x)$ is an interval fuzzy set determined by two final outputs related with the left and the right part of primary membership functions which are called as $Y_{L}(x)$ and $Y_{R}(x)$ respectively. They can be expressed by the following Eqs. (16) and (17) as:

$$
Y_{L}=\frac{\sum_{l=1}^{l e} \Theta_{l} \overline{v_{1}}(x)}{\sum_{l=1}^{l e} \overline{v_{1}}(x)}+\frac{\sum_{l=l e}^{M} \Theta_{l} \underline{v}_{1}(x)}{\sum_{l=l e}^{M} \underline{v}_{1}(x)}
$$

and

$$
Y_{R}=\frac{\sum_{l=1}^{l e} \Theta_{l} \underline{v}_{1}(x)}{\sum_{l=1}^{l e} \underline{v}_{1}(x)}+\frac{\sum_{l=r i}^{M} \Theta_{l} \bar{v}_{1}(x)}{\sum_{l=r i}^{M} \underline{v}_{1}(x)}
$$

where $\Theta_{j}$ is nothing but the weight of fuzzy set which should be associated with the 1-th rule, $j=1, \cdots, M$, le is the rule that exchange the UMF to LMF, $r i$ is the rule that exchange the LMF to UMF. $\bar{v}(x)$ and $\underline{v}(x)$ are calculated as following Eqs. (18) and (19) 


$$
\bar{v}(x)=\bar{\mu}_{\tilde{F}_{1}^{j}}\left(x_{1}\right) * \cdots * \bar{\mu}_{\tilde{F}_{p}^{j}}\left(x_{p}\right)
$$

and

$$
\underline{v}(x)=\underline{\mu}_{\tilde{F}_{1}^{j}}\left(x_{1}\right) * \cdots * \underline{\mu}_{\tilde{F}_{p}^{j}}\left(x_{p}\right)
$$

To determine the final output $Y_{L}(x)$, from the Eq. (20), it is necessary to determine

$v_{l L}=\frac{\overline{v_{l}}(x)+\underline{v}_{l}(x)}{2}$

We first consider the upper $\mathrm{mf}(U M F)$ in the primary rule $l=1$ to $l e$. After that, we have exchanged $U M F$ by $L M F$, considering now $l=l e$ to $M$. To determine this specific rule $l=l e$ and to calculate the final output $Y_{L}(x)$. In this procedure, the first calculus of $Y_{L}(x)$ is given by Eq. (21)

$$
Y_{L}=\frac{\sum_{l=1}^{M} \Theta_{l} v_{l L}(x)}{\sum_{l=1}^{M} v_{l L}(x)}
$$

By semilunar procedure, the calculus of $Y_{R}(x)$ is given by Eq. (22)

$$
Y_{R}=\frac{\sum_{r=1}^{M} \Theta_{r} v_{r R}(x)}{\sum_{r=1}^{M} v_{r R}(x)}
$$

However, the rule le is exchanged by $r i$, and (23) is evaluated instead of Eq. (20). Finally, the defuzzification of $I T 2-F L S$ is given by

$$
Y(x)=\frac{Y_{L}(x)+Y_{R}(x)}{2}
$$

which $Y(x)$ is the defuzzified value of IT2F variables.

\section{Motivation and model formulation}

\subsection{Motivation}

Fire monitoring in UCMs are much more flat to natural fire occurrences than other solid mineral mines. Coal being a costly mineral, the damage and destruction by fire in the UCMs are also catastrophic. The coals in underground mines are subject to fire by natural oxidation procedure, and when caught on fire, it is quite nearly not possible to reduce the spread, if the fire is not detected and prevented at the early stage. So, it is quite important to sense fire at a stage that is close to its initiation. The underground structures of different mines are different. It is not easy to work in UCMs imprecise environments with limited resources and capabilities. Especially coal mines are much more unsafe with respect to fire catching and presence of explosive gas and dust. wireless sensors which are capable of detecting fire can be used to monitor and send alarm automatically to the DM. These come really useful in that situation. Various low-power and cost-effective sensor platforms have been developed based upon recent advances in wireless communication and micro system technologies. During this unstructured combustion process, the combustion gases like $\mathrm{CO}$ and $\mathrm{CO}_{2}$ are mainly produced as compared to the hydrocarbons with reduction of $\mathrm{O}_{2}$ and liberation of heat. The enlarge in temperature and concentrations of $\mathrm{CO}$ and $\mathrm{CO}_{2}$ gases in UCM imprecise environment not only gives definitive early indication of the occurrence of fire due to impulsive combustion but also the status of fire as well. There are various fire indices are used for early detection and assessing the status of fire in underground coal mines. Among these, Graham's ratio, Young's ratio and oxides of carbon ratio $\left(\mathrm{CO} / \mathrm{CO}_{2}\right)$ are most commonly used in practice. This is because fire in underground coal mines is largely caused due to spontaneous combustion of coal. Hence, in our type-2 fuzzy model, we used temperature, $\mathrm{CO}, \mathrm{CO}_{2}$ and $\mathrm{O}_{2}$ as the input parameters for early detection of fire in UCMs.

Thus, in order to establish a safety measure for mines, The WSNs can prove to be useful and reliable prediction in an imprecise data. There are two types of WUSN based coal mine monitoring system has been deployed (a) Long wall mine in Fig. 1, which is formed of UMC where a long wall of coal is mined in a single slice and (b) Bord and pillar mine in Fig. 2, where the mined material is extracted across a horizontal plane, creating horizontal arrays of rooms and pillars.

WUSN has been developed for the observation of environmental conditions measuring the major parameters responsible for its production in a regular interval of the mines and to alert early to the ground monitoring system at the time of risk (cf. Akyildiz and Stuntebeck 2006). In this case, alert signal is generated when the responsible environmental parameters gets more than the threshold value and this type of system is very unpredictable and complex for observation since, the alarming signals are never generated by the system despite the nearer value of monitoring data to threshold value. To improve this type of problem, T2FLC requires to be incorporated to WUSN for designing of an efficient as well as trustworthy monitoring system in UCM (Fig. 3).

\subsection{Model formulation}

During this spontaneous combustion process in a coal mines, the combustion gases like $\mathrm{CO}$ and $\mathrm{CO}_{2}$ are primarily formed as compared to the hydrocarbons with depletion of $\mathrm{O}_{2}$ and release of heat and combining these parameters, we formulate a block diagram of proposed model in Fig. 4. The increment in temperature and 


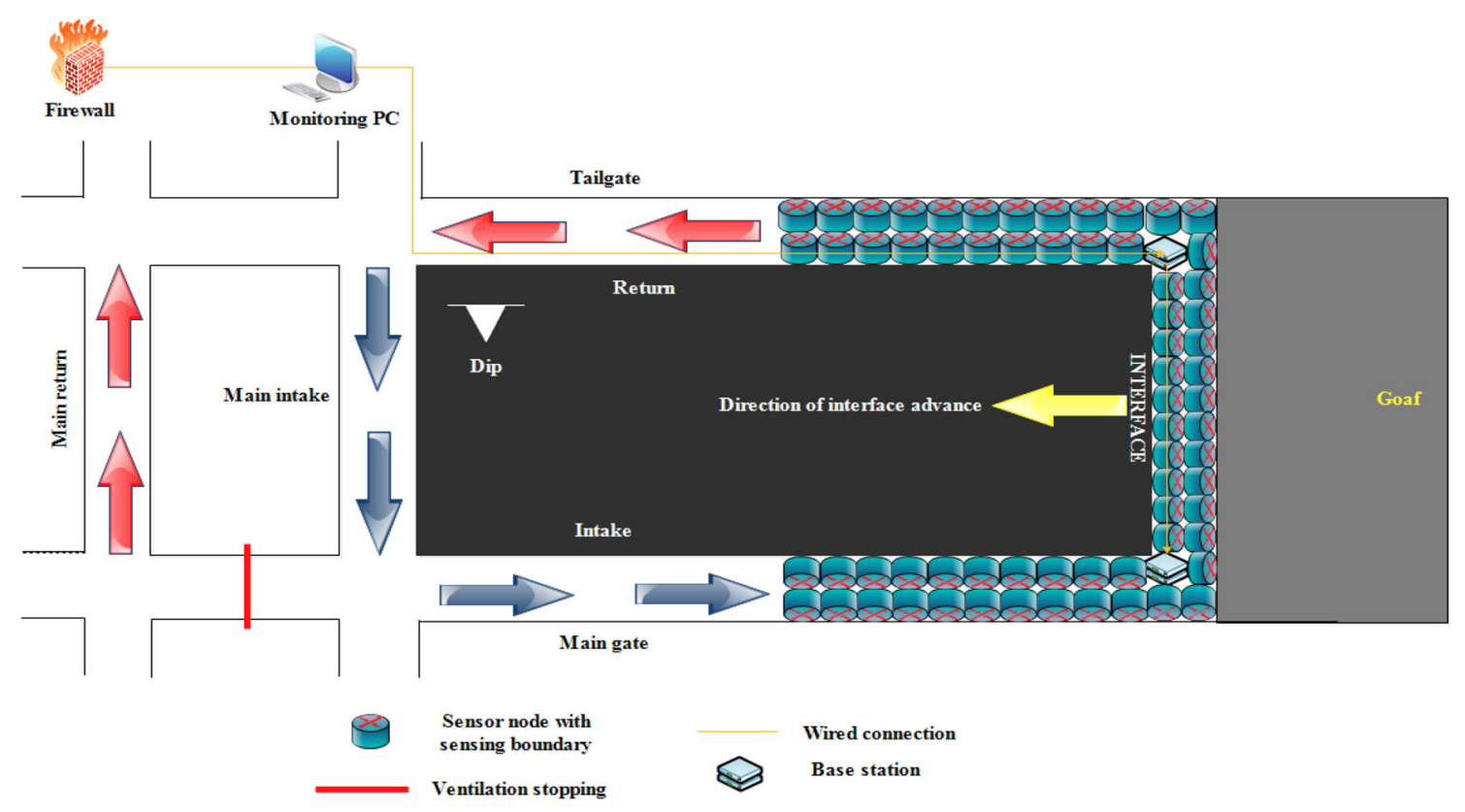

Fig. 1 WUSN based coal mine monitoring system deployed in Long-wall mine

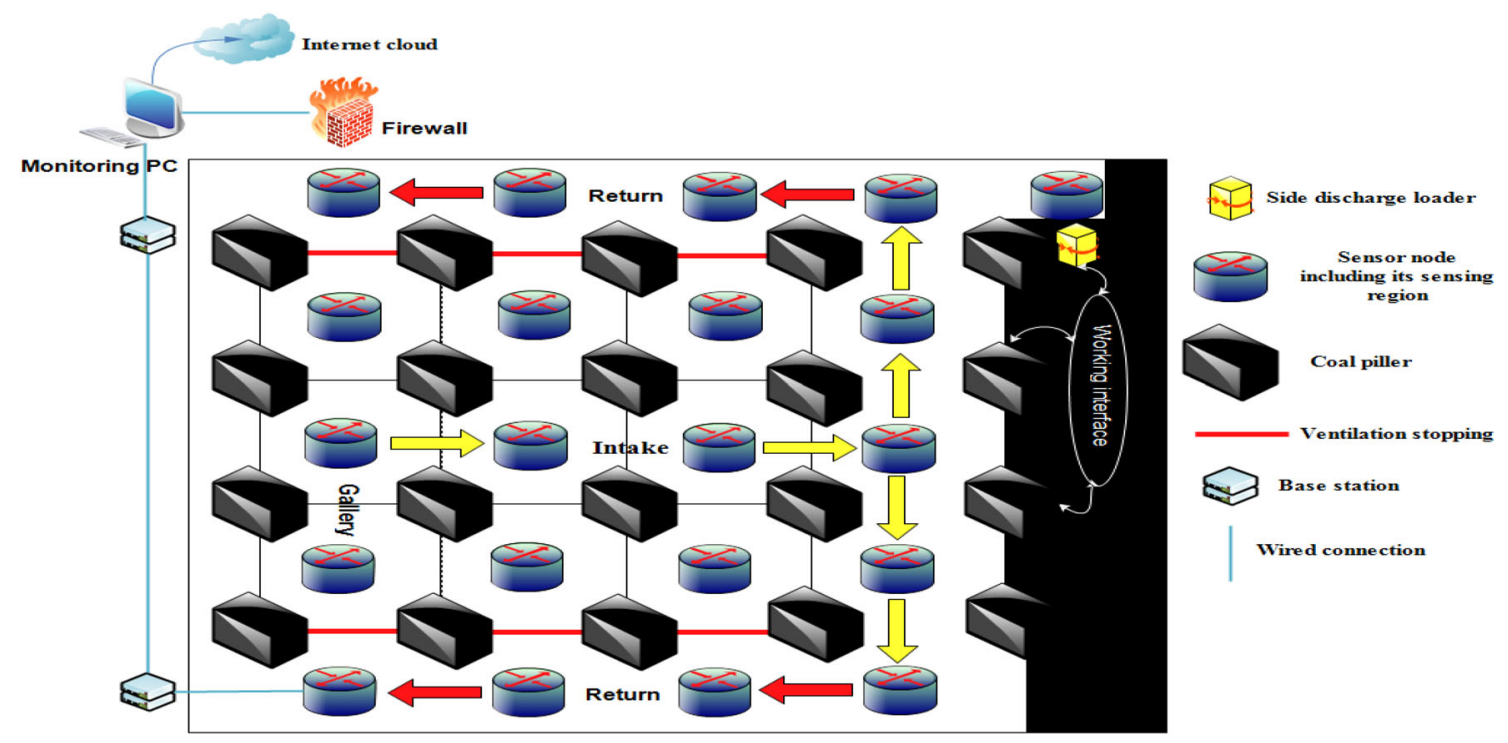

Fig. 2 WUSN based coal mine monitoring system deployed in Bord and pillar mine

concentrations of $\mathrm{CO}$ and $\mathrm{CO}_{2}$ gases in UCM environment gives definitive early indication of the occurrence of fire due to spontaneous combustion too the status of fire as well. Therefore, in our T2F model, we have utilized temperature, $\mathrm{CO}, \mathrm{CO}_{2}$ and $\mathrm{O}_{2}$ as the input parameters can be used for early detection of fire in UCM. Their MFs have been shown in Figs. 5, 6, 7, 8 respectively and one output parameter fire intensity that MF has shown in Fig. 9. The fire monitoring system in UCM based on T2FLC approach is presented in Fig. 2. Based on practical data set, we have formulated T2F rules in Fig. 11. The flowchart for the proposed T2FIS model has been depicted in Fig. 10.

\section{Solution procedure}

In the first stage, we have collected source data and calculated input criteria for the proposed inference model. According to previously adopted assumptions, the basic criteria for assessing operational risk assessment in coal mining enterprisers are based on practical data, which may 


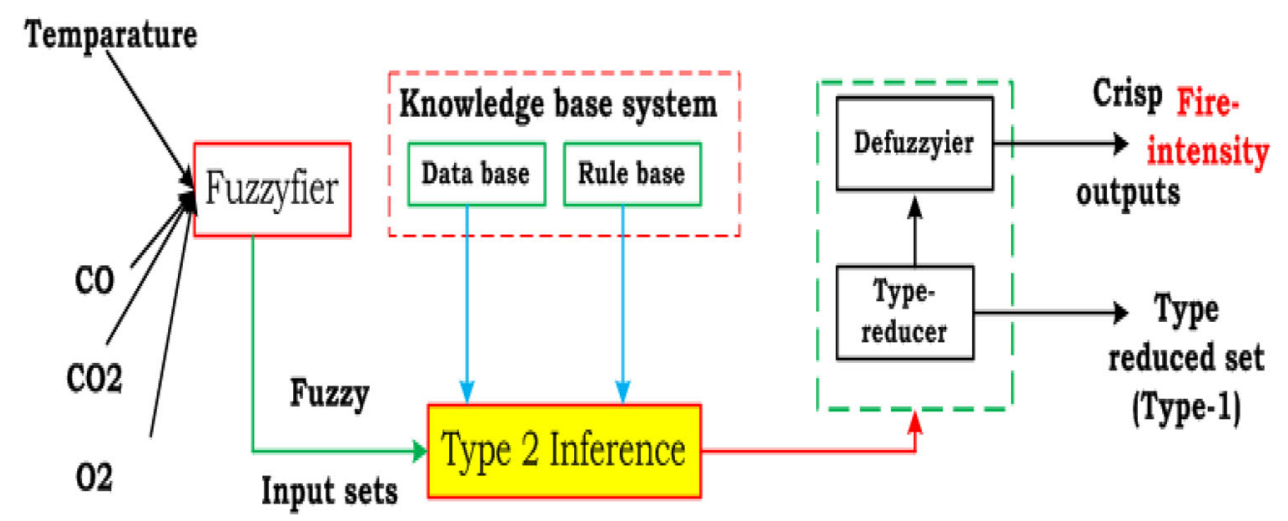

Fig. 3 The structure of a IT2FLS

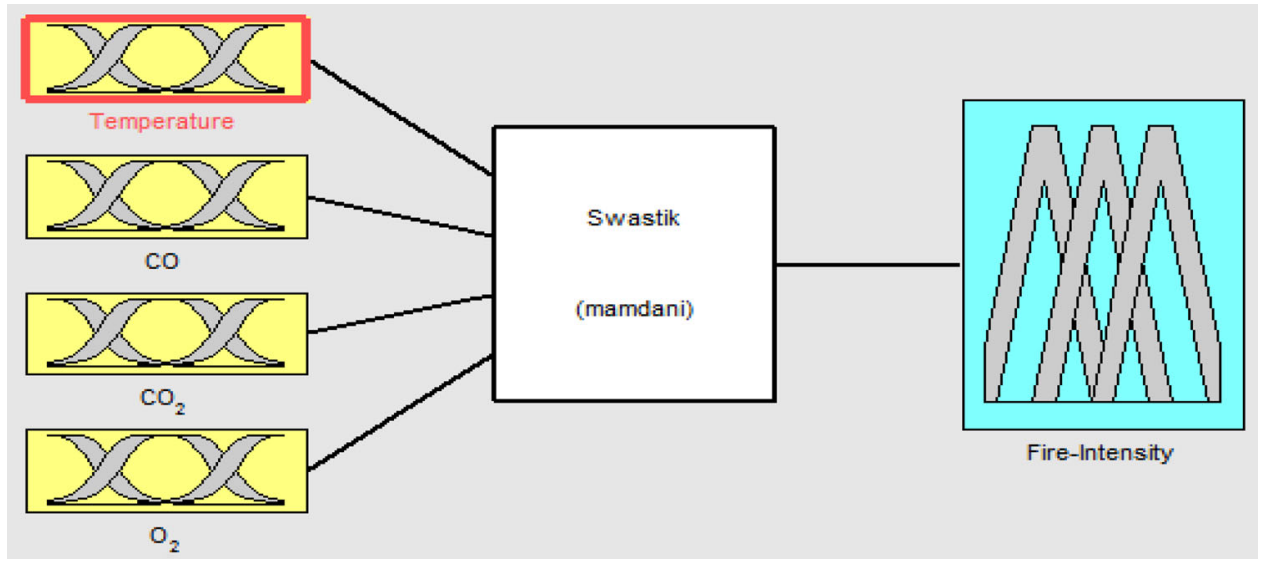

Fig. 4 Block diagram for IT2FIS

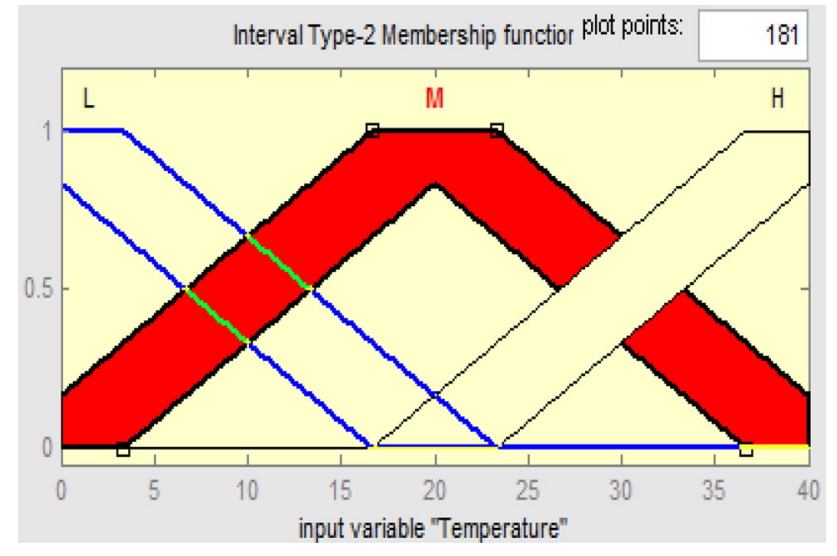

Fig. 5 MFs for temperature

be obtained by analyzing the content of interim reports and financial statements by the mining companies.

In the second stage, for the needs of the "fuzzification" module, we have determined the form of the type-2 fuzzy sets for respective four input variables as $\mathrm{CO}, \mathrm{CO}_{2}, \mathrm{O}_{2}$ and temperature, and also for these variables the basic terms set and the division of their value spaces. Due to similarities in

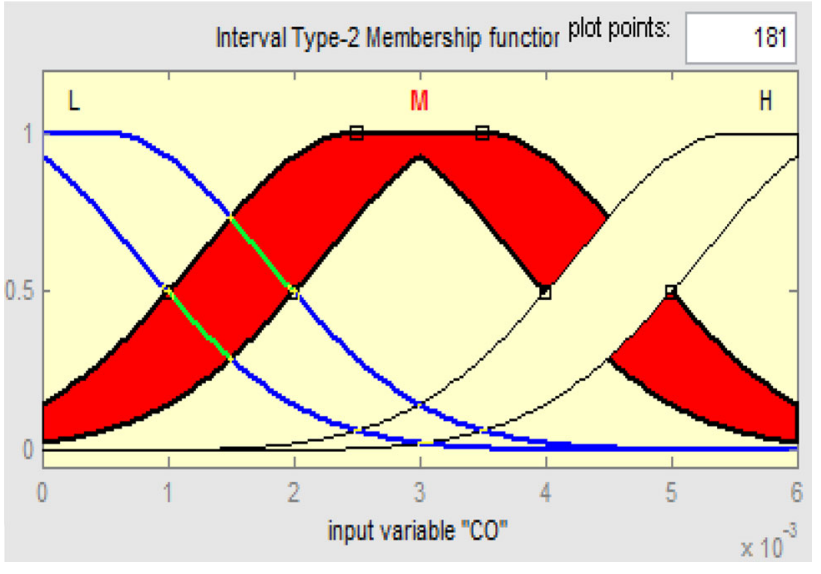

Fig. 6 MFs for carbon monoxide

the expression of natural language assessments of the individual variables, for the purpose we have developed a type-2 fuzzy (T2F) model. We have adopted the same linguistic vocabulary and division of values space for the respective groups input and output variables. We considered in the case of input variables, into three T2F membership functions (mfs) \{low, medium, high $\}$ and for 


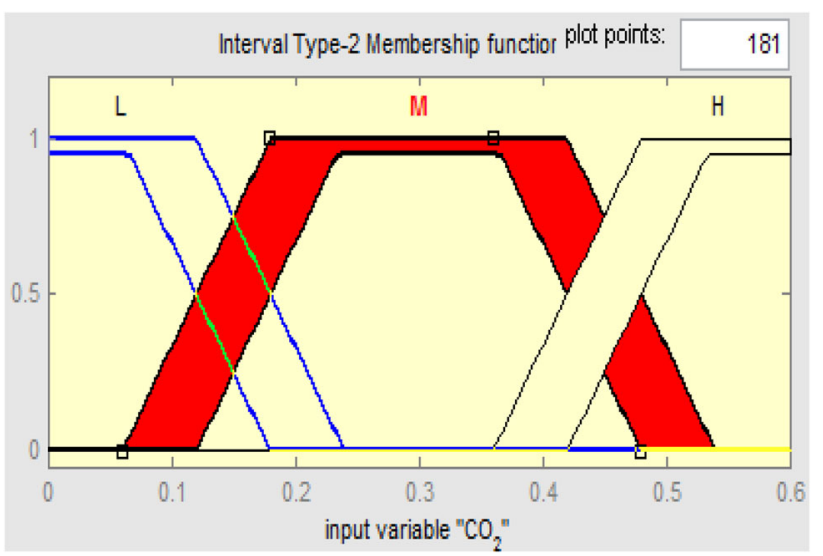

Fig. $7 \mathrm{MFs}$ for $\mathrm{CO}_{2}$

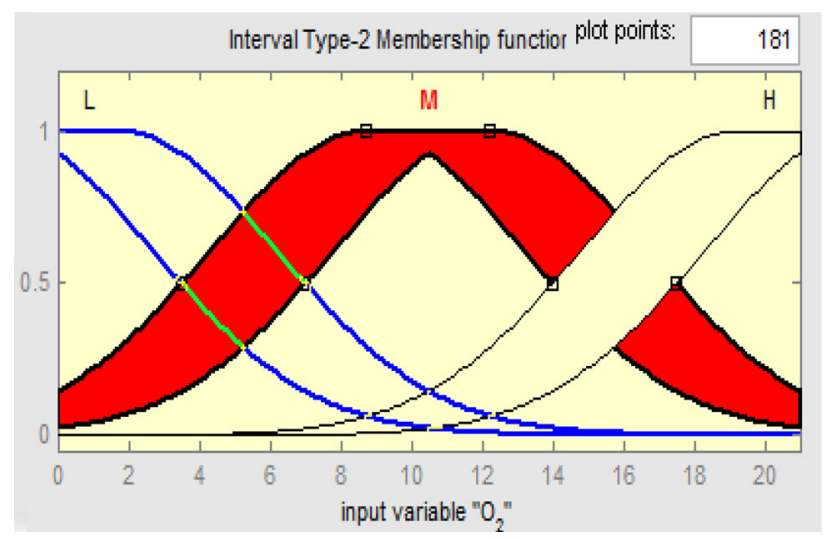

Fig. $8 \mathrm{MFs}$ for $\mathrm{O}_{2}$

output variable fire intensity, in order to obtain more accurate results, into five fuzzy $\mathrm{mfs}\{$ Very-low, low, medium, high, very-high $\}$.

We propose a WSN-based fire monitoring system for UCMs using type-2 fuzzy logic approach that fuzzy inference system in uncertain environment. The SNs are deployed in the UCMs for collecting the monitoring data, such as temperature and concentrations of various gases like $\mathrm{CO}, \mathrm{CO}_{2}$ and $\mathrm{O}_{2}$. These data are input to the type-2 fuzzy system which in turn produces the status of fire in UCMs. The type-2 fuzzy system is implemented at sensor nodes and base station (sink) for making realtime decisions from the monitored imprecise data. The main objective of the proposed research work is to minimize the delay in decision-making process and provide an efficient and reliable fire monitoring system for underground coal mines. Recently, Grychowski (2014) has proposed an offline fire monitoring system for underground coal mines using fuzzy inference system. It measures the performance of type-1 fuzzy based fire monitoring system by using previously collected monitoring data which is a delayed network and not responsive to the imminent hazards.

Moreover, because of the ease of use and great versatility of both variable groups, we adopted the trapezoidal shape of the mfs for each type-2 fuzzy set (cf. Jana and Ghosh 2018; Jana et al. 2017, 2019) and equal division of their value spaces (Figs. 5-8). The characteristic points describing the mfs of each type-2 fuzzy set for four input variables were set arbitrarily based on the distribution of analyzed variable values. We have created a relevant type2 fuzzy inference rules for the needs of the "inference" module, determining the mechanism of inference and defining the one output membership function of the proposed inference model. Due to the lack of proper information, knowledge and experience in this area of coal mining, the respective rules are elaborated without the use of external experts. Taking into consideration the inference model structure (Fig. 9), We have formulated 18 knowledge base rule in the form of IF-THAN in Fig. 11.

\section{Results and discussion}

\subsection{Statistical data analysis}

We have formulated a model for improving fire intensity assessment w.r.t. each four input and one output imprecise

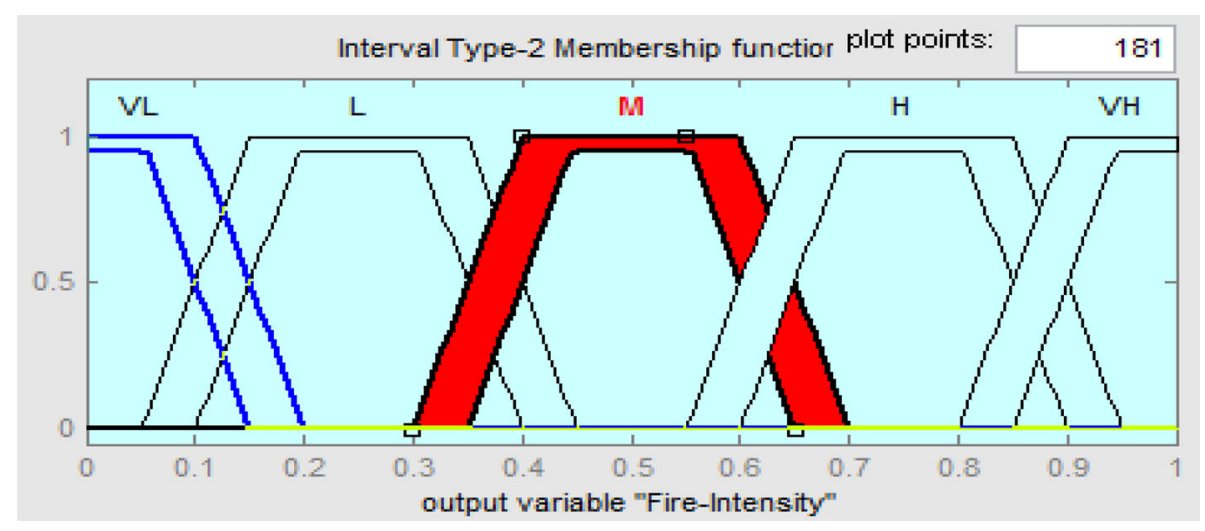

Fig. 9 MFs for output put parameter fire intensity 


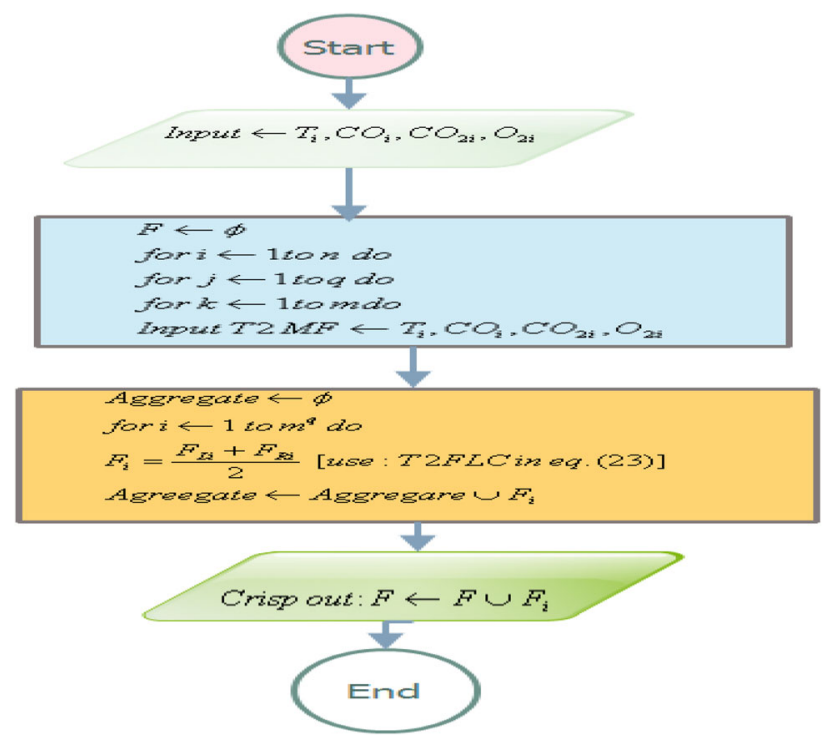

Fig. 10 Flowchart of the proposed T2FIS

data of coal mines security using T2FLC in fuzzy environment. In order to control the fire intensity of the prepared model, some statistical parameters have been defined. The prediction capability of proposed fire intensity model of coal mines security is evaluated by using the test data in the trained data and comparing the outputs and measured values. In addition, the statistical parameters such as the root mean square error (RMSE) and the determination coefficient $\left(R^{2}\right)$ are used to compare predicted and measured values of flexible modulus.

The RMSE is defined by the following Eq. (24):

$R M S E=\sqrt{\frac{1}{n} \sum_{i=1}^{n}\left(x_{\text {pred }_{i}}-x_{o b s_{i}}\right)^{2}}$

In addition, the determination coefficient $\left(R^{2}\right)$ can be calculated using Eq. (25) that is calculated by:

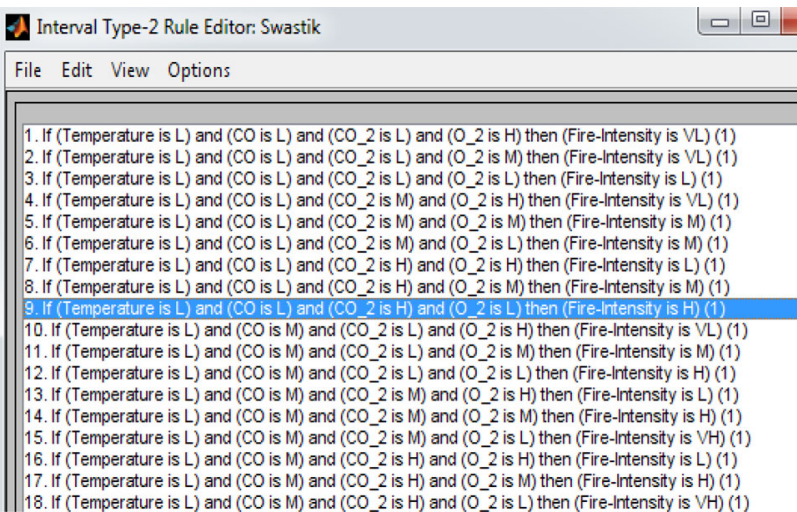

Fig. 11 Rule for T2FIS
$R^{2}=1-\frac{\sum_{i=1}^{n}\left(x_{p r e d}-x_{o b s_{i}}\right)^{2}}{\sum_{i=1}^{n} x_{o b s_{i}}^{2}}$

The mean absolute percentage error (MAPE) measures the average of the squares of the errors. The smaller values of MAPE ensure the better performance of the proposed models. The MAPE is calculated by the following Eq. (26):

MAPE $=\frac{1}{n} \sum_{i=1}^{n} \frac{\left|\left(x_{\text {pred }_{i}}-x_{\text {obs }_{i}}\right)\right|}{x_{\text {pred }_{i}}} \times 100 \%$

The performance efficiency of the proposed models is also evaluated with the help of mean absolute error (MAE), which can be defined by the Eq. (27):

$M A E=\frac{1}{n} \sum_{i=1}^{n}\left|x_{\text {pred }_{i}}-x_{o b s_{i}}\right|$

where $n$ is the number of data patterns in the data set, $x_{\text {pred }_{i}}$ defines the predicted value of one data point $i$ and $x_{o b s_{i}}$ is the observed value of one data point $i$. In Table 2, a statistical data analysis of output has shown.

\subsection{Multiple linear regression for error analysis}

If $x_{i}$ equals to process parameters and $e_{i}$ denotes low predictive error that is optimization target for coal mine fire hazard capturing capability, the Multiple Linear Regression (MLR) can be written as

$y_{i}=b_{0}+b_{1} x_{i 1}+b_{2} x_{i 2}+\cdots+b_{n} x_{i n}+e_{i}$

Several quality measuring parameters should be used to understand the performance efficiency and accuracy of the proposed models and this is accomplished by carrying out statistical error analysis. To evaluate and compare the performance efficiency and accuracy of the proposed model with the standard data, the most common statistical quality measures. The RMSE (i.e. root mean squared error) and correlation coefficient $\left(R^{2}\right)$ have been employed by using regression in Matlab 14 and shown in Table 3.

Table 2 Statistical data analysis for T2 and T1FLC

\begin{tabular}{lllll}
\hline FIS & RMSE & $R^{2}$ & MAPE & MAE \\
\hline T2FLC & 0.104 & 0.991 & 4.703 & 0.072 \\
T1FLC & 0.056 & 0.953 & 3.965 & 0.039 \\
\hline
\end{tabular}

Table 3 Statistical analysis of output data using ANFIS and MLR

\begin{tabular}{llllll}
\hline Model & Approach & \multicolumn{2}{l}{ Statistics } & & \\
\cline { 3 - 6 } & & RMSE & MAE & MAPE & $R^{2}$ \\
\hline 1 & MLR & 0.05313 & 0.0752 & 0.968 & 0.9714 \\
& ANFIS & 0.05387 & 0.0763 & 0.978 & 0.9811 \\
\hline
\end{tabular}


It is observed that the proposed model is consistence and it can be implemented for fire monitoring at coal mine via WUSN.

\section{Simulation results and discussion}

Data collected from UCM (temperature, carbon mono-oxide and oxygen) are simulated using Mamdani IT2FIS. From the collected data, it can be witnessed on the increment in temperature, $\mathrm{CO}$ and $\mathrm{CO}_{2}$ concentrations, while the concentration of oxygen decreases w.r.t. time. This occurs due to the spontaneity of the combustion process of coal. The Mamdani IT2FS receives these data and generates the fire intensity model on the basis of data characteristics as output which are shown in Figs. 12, 13, 14, 15. The steps involved for simulation in resulting the fire intensity of UCM from collected data using fuzzy logic are shown in Fig. 9. from which should be modified as the logical approach is of four steps. It converts the collected data into T2F input mfs. Then these inputs were evaluated using the Mamdani fuzzy rules given in Fig. 11. Then the T2F outputs were combined into a single output like fire

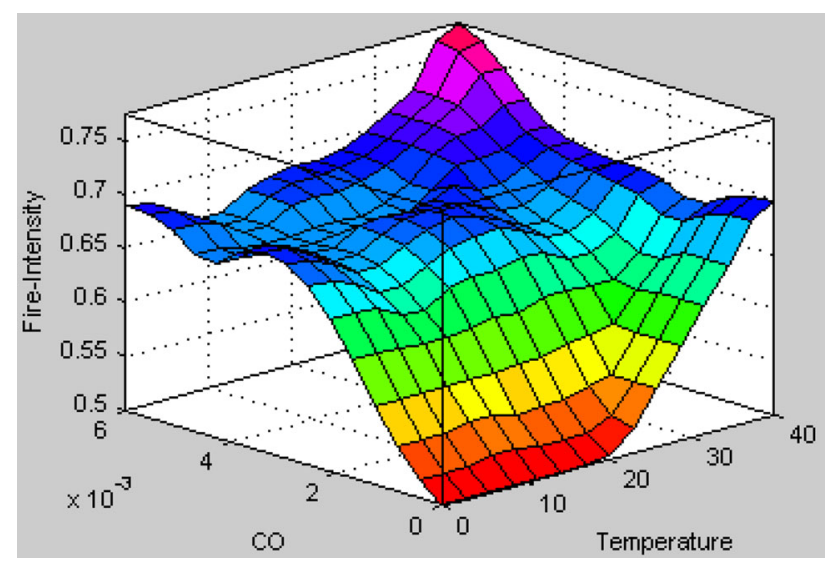

Fig. 12 Change of FI w.r.to temperature, CO

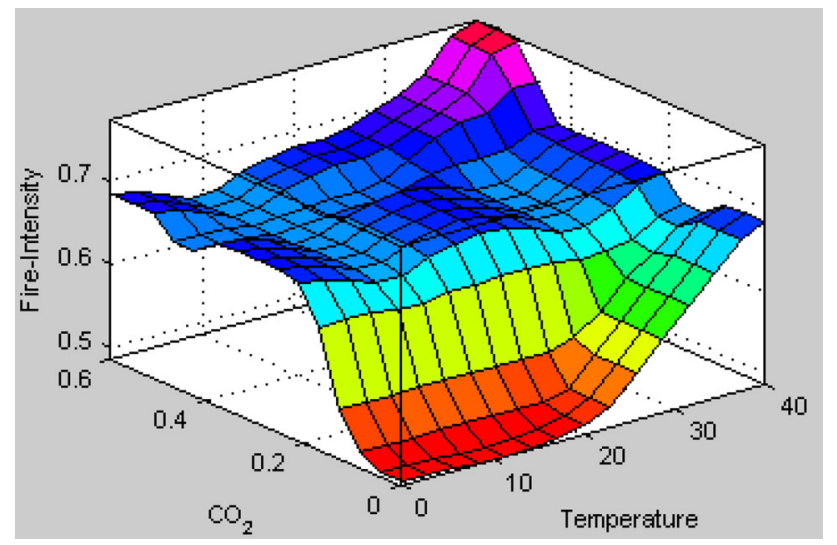

Fig. 13 Change of FI w.r.to temperature, $\mathrm{CO}_{2}$

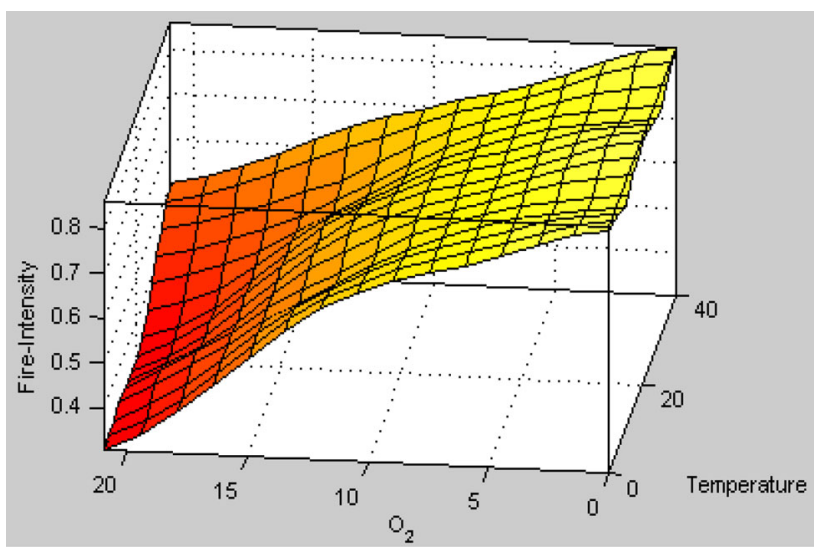

Fig. 14 Change of FI w.r.to temperature, $\mathrm{O}_{2}$

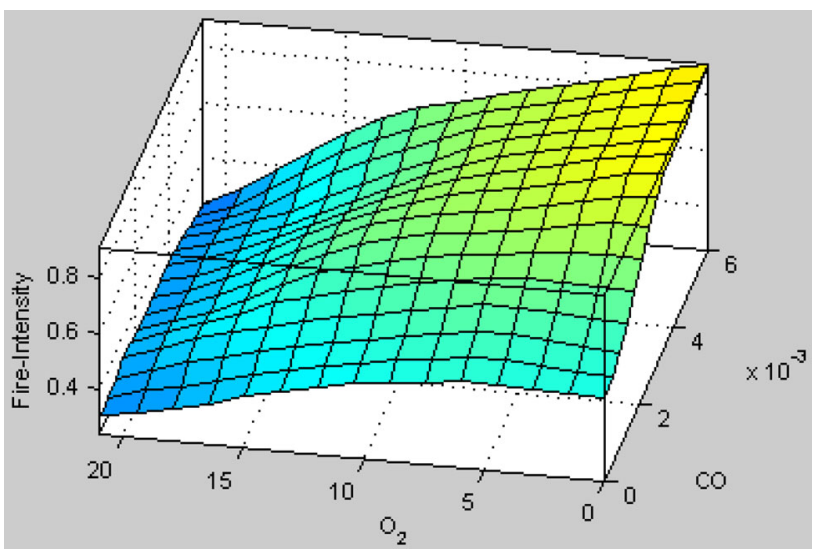

Fig. 15 Change of FI w.r.to $\mathrm{O}_{2}$, $\mathrm{CO}$

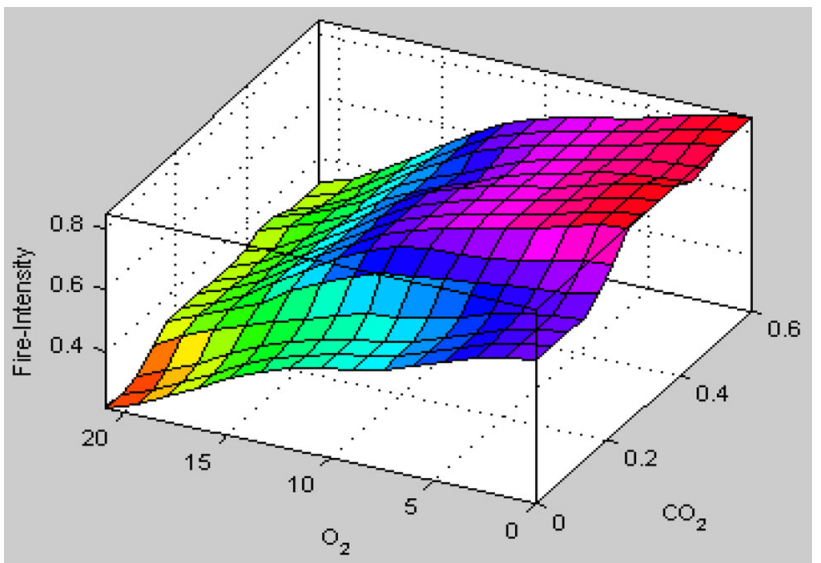

Fig. 16 Change of FI w.r.to $\mathrm{O}_{2}, \mathrm{O}_{2}$

intensity. At last, fire intensity is displayed as a crisp output using COG defuzzification method (Figs. 16, 17). Out of 35 sets of data collected, 10 sets of randomly selected data and the indices including $R^{2}, R M S E$, were used to evaluate the performance of model. The results show that for 10 sets of randomly selected data, the type-2 fuzzy logic model, with $R^{2}=0.99, R M S E=2.10$, performs better than the 


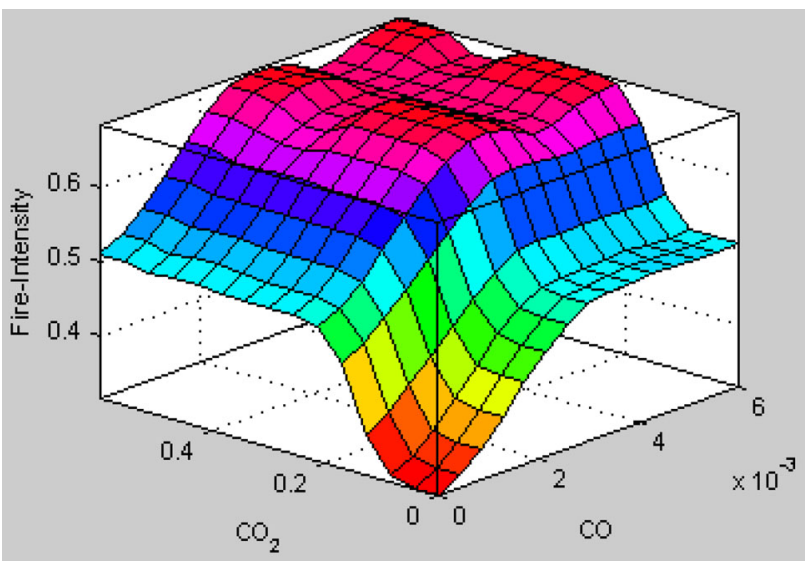

Fig. 17 Change of FI w.r.to $\mathrm{CO}_{2}$, $\mathrm{CO}$

multiple regression models and may be used for the estimation in the longwall panels. This paper provides a new way to support fire hazard assessment in a preliminary concept in type-2 fuzzy environment and this problem requires further research followed by verification in industry. The regression lines have been drawn for T1FIS in Fig. 18 and T2FIS in Fig. 19 respectively. From these result T2FIS has provided better performance which is expect by coal mine manager.

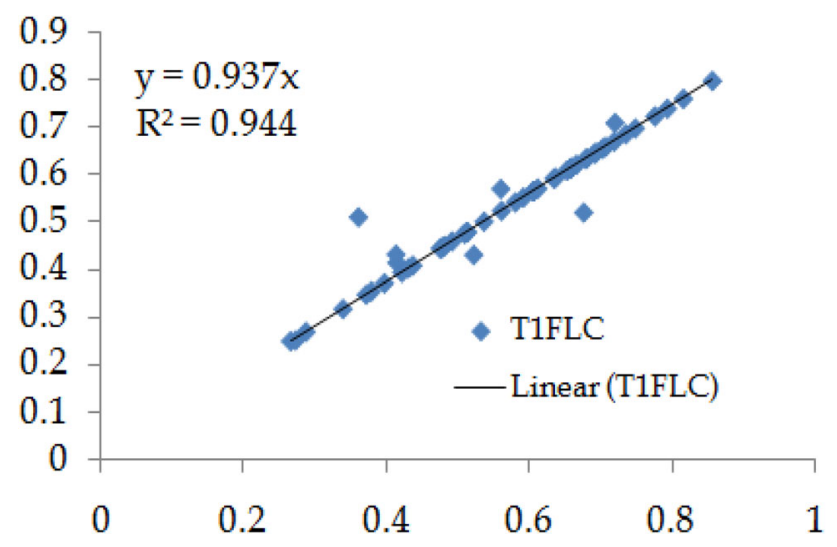

Fig. 18 Value of $R^{2}$ w.r.t IT1FIS

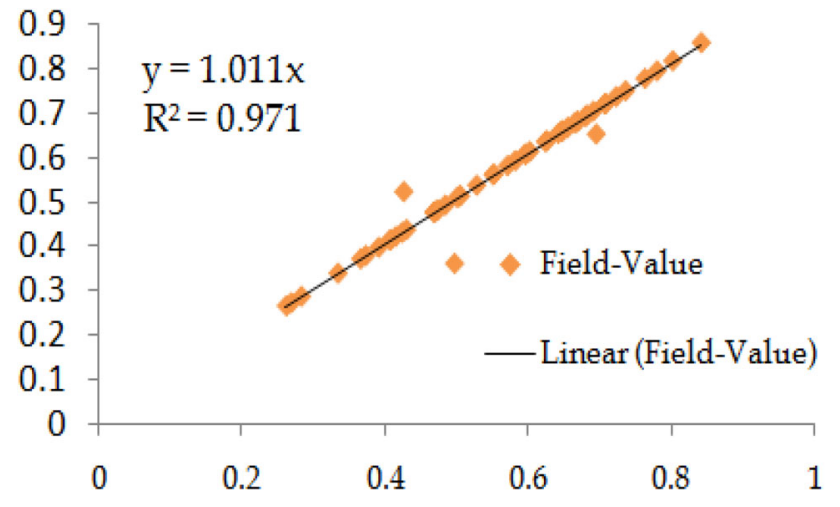

Fig. 19 Value of $R^{2}$ w.r.t IT2FIS

\section{Conclusions and future research work}

In this paper, we have developed interval type-2 fuzzy logic control approach for evaluation of prompt sensing capability of any fire risk situation and to handle the safety assessment in UCM through the implementation of WUSN system. The T2FLC helped by assisting to trace the inputs and outputs in a well-secured as well as comprehensive manner for developing the inferences train so that various types of underground environmental conditions could be predicted during coal mining from UCM system. The help of this particular T2FLC is taken for the implementation of WUSN because the difficulty and complexity both are higher to take decisions from ground monitoring system through wired connection including the high uncertainty and rough nature of underground. Prediction of various types of environmental conditions helps to optimize the sensing parameters during the underground monitoring system of UCM. The authors argue that the suggested T2FLC method is a completely new and unique approach for construction and controlling the WUSN of UCM during coal mining proposed in this paper needs to be developed, regarding coal mining from UCM that must be satisfied to get an effective output in the workplace. Moreover, in this paper, the financial effects of the discussed process are not taken into account while constructing an interval type- 2 fuzzy rule base. The study is mainly focused on the development of WUSN system in UCM for the safety assessment to minimize the fire risk hazard in adverse condition. Therefore, quantitative factors responsible to get positive output can be easily included in the fuzzy prediction model for improving accuracy. The major contribution of this research work is proposing a systematic integrated approach for modelling process condition and their prediction so that accuracy of sensor and its performance efficiency during coal mining in UCM can be improved. For further research work, we will utilize and integrate other intelligent methods, such as interpretative structural modelling method, simulink, fuzzy artificial neural network, to evaluate the scale of efficiency of our current work.

\section{Compliance with ethical standards}

Conflict of interest The authors have no conflict of interest for the publication of this paper.

Open Access This article is distributed under the terms of the Creative Commons Attribution 4.0 International License (http://crea tivecommons.org/licenses/by/4.0/), which permits unrestricted use, distribution, and reproduction in any medium, provided you give appropriate credit to the original author(s) and the source, provide a link to the Creative Commons license, and indicate if changes were made. 


\section{References}

Akyildiz IF, Stuntebeck EP (2006) Wireless underground sensor networks: research challenges. Ad Hoc Netw 4(6):669-686

Banerjee SC (1985) Spontaneous combustion of coal and mine fires. Balkema, Rotterdam

Bhattacharjee S, Roy P, Ghosh S, Misra S, Obaidat MS (2012) Wireless sensor network-based fire detection, alarming, monitoring and prevention system for Bord-and-Pillar coal mines. J Syst Softw 85(3):571-581

Castillo O, Melin P (2008) Type-2 fuzzy logic: theory and applications, studies in fuzziness and soft computing, vol 223. Springer, Berlin

Cheng B, Cheng X, Chen J (2015) Lightweight monitoring and control system for coal mine safety using REST style. ISA Trans 54:229-239

Dey S, Jana DK (2016) Application of fuzzy inference system to polypropylene business policy in a petrochemical plant in India. J Clean Prod 112:2953-2968

Grychowski T (2014) Multi sensor fire hazard monitoring in underground coal mine based on fuzzy inference system. J Intell Fuzzy Syst 26(1):345-351

Jana DK, Ghosh R (2018) Novel interval type-2 fuzzy logic controller for improving risk assessment model of cyber security. J Inf Secur Appl 40:173-182

Jana DK, Bej B, Wahab HM, Mukherjee A (2017) Novel type-2 fuzzy logic approach for inference of corrosion failure likelihood of oil and gas pipeline industry. Eng Fail Anal 80:299-311

Jana DK, Roy K, Dey S (2019) Comparative assessment on lead removal using micellar-enhanced ultrafiltration (MEUF) based on a type-2 fuzzy logic and response surface methodology. Sep Purif Technol 207:28-41

Liu Z, Li C, Wu D, Dai W, Geng S, Ding Q (2010) A wireless sensor network based personnel positioning scheme in coal mines with blind areas. Sensors 10(11):9891-9918

Mamdani EH, Assilian S (1975) An experiment in linguistic synthesis with a fuzzy logic controller. Int J Man-Mach Stud 7:1-13

Mishra DP, Sugla M, Singha P (2013) Productivity improvement in underground coal mines: a case study. J Sustain Min 12(3):48-53

Muduli Lalatendu, Mishra Devi Prasad, Jana Prasanta K (2018) Application of wireless sensor network for environmental monitoring in underground coal mines: a systematic review. J Netw Comput Appl 106:48-67

NawrockiIzabela TL, Kowalska J (2016) Assessing operational risk in coal mining enterprises-Internal, industrial and international perspectives. Resources Policy 48:50-67

Rawat R, Singh KD, Chaouchi Hakima, Bonnin Jean Marie (2014) Wireless sensor networks: a survey on recent developments and potential synergies. J Supercomput 68(1):1-48

Sugeno, M (1985) Industrial applications of fuzzy control. Amsterdam, New York, NY, USA: North-Holland. Sole distributors for the U.S.A. and Canada, Elsevier Science Publishing company

Xu X, Zheng P, Li L, Chen H, Ye J, Wang J (2012) Design of underground miner positioning system based on zigbee technology Web Information Systems and Mining. Springer, Berlin, pp 342-349

Zadeh LA (1965) Fuzzy sets. Inf Control 8:338-353

Zhi-zhong J, Simplot-Ryl D, Leung Victor CM (2010) Ad hoc networks: second international conference, ADHOCNETS 2010, Victoria, BC, Canada, Springer, p 49 